\title{
Drift chambers for a precision measurement of the Michel parameters in muon decay
}

Yu. Davydov*,a,b, W. Andersson ${ }^{\mathrm{a}}$, M. Comyn ${ }^{\mathrm{a}}$, P. Depommier ${ }^{\mathrm{c}}$, J. Doornbos $^{\mathrm{a}}$, W. Faszer ${ }^{\mathrm{a}}$, C.A. Gagliardi ${ }^{\mathrm{d}}$, D.R. Gill ${ }^{\mathrm{a}}$, P. Green ${ }^{\mathrm{e}}$, P. Gumplinger ${ }^{\mathrm{a}}$, J.C. Hardy ${ }^{\mathrm{d}}$, M. Hasinoff ${ }^{f}$, R. Helmer ${ }^{\mathrm{a}}$, R. Henderson ${ }^{\mathrm{a}}$, A. Khruchinsky ${ }^{\mathrm{b}}$, P. Kitching ${ }^{\mathrm{e}}$, D.D. Koetke ${ }^{\mathrm{g}}$, E. Korkmaz ${ }^{\mathrm{h}}$, Yu. Lachin ${ }^{\mathrm{b}}$, D. Maas ${ }^{\mathrm{f}}$, J.A. Macdonald ${ }^{\mathrm{a}}$, R. MacDonald ${ }^{\mathrm{e}}$, R. Manweiler ${ }^{\mathrm{g}}$, G. Marshall ${ }^{\mathrm{a}}$, E.L. Mathie ${ }^{\mathrm{i}}$, L. Miassoedov ${ }^{\mathrm{b}}$, J.R. Musser ${ }^{\mathrm{d}}$, P. Nord ${ }^{\mathrm{g}}$, A. Olin ${ }^{\mathrm{a}}$, R. Openshaw ${ }^{\mathrm{a}}$, D. Ottewell ${ }^{\mathrm{a}}$, T. Porcelli ${ }^{\mathrm{h}}$, J-M. Poutissou ${ }^{a}$, R. Poutissou ${ }^{\mathrm{a}}$, G. Price ${ }^{\mathrm{i}}$, M.A. Quraan ${ }^{\mathrm{e}}$, N.L. Rodning, J. Schaapman ${ }^{\mathrm{e}}$, V. Selivanov ${ }^{\mathrm{b}}$, G. Sheffer ${ }^{\mathrm{a}}$, B. Shin ${ }^{j}$, F. Sobratee ${ }^{\mathrm{e}}$, J. Soukup ${ }^{\mathrm{e}}$, T.D.S. Stanislaus ${ }^{\mathrm{g}}$, G. Stinson ${ }^{\mathrm{e}}$, R. Tacik ${ }^{\mathrm{i}}$, V. Torokhov ${ }^{\mathrm{b}}$, R.E. Tribble ${ }^{\mathrm{d}}$, M.A. Vasiliev ${ }^{\text {d, H.C. Walter }}{ }^{\mathrm{a}}$, D. Wright ${ }^{\mathrm{a}}$

${ }^{a}$ TRIUMF, Vancouver, BC, V6T 2A3. Canada

${ }^{\mathrm{b}}$ RRC "Kurchatov Institute", Moscow, Russia

${ }^{\mathrm{c}}$ University of Montreal, Montreal, Que., Canada

${ }^{\mathrm{d}}$ Texas A\&M University, College Station, TX, USA

${ }^{\mathrm{e}}$ University of Alberta, Edmonton, Alta., Canada

${ }^{\mathrm{f}}$ University of British Columbia, Vancouver, BC, Canada

${ }^{g}$ Valparaiso University, Valparaiso, IN, USA

${ }^{\mathrm{h}}$ University of Northern British Columbia, Prince George, BC, Canada

${ }^{\mathrm{i}}$ University of Regina, Regina, Sask., Canada

${ }^{\mathrm{j}}$ University of Saskatchewan, Saskatoon, Sask., Canada

\section{Abstract}

Planar drift chambers will be used in a solenoidal magnetic field to measure the Michel parameters in muon decay. The chamber manufacture procedure uses flat glass tables and precise ceramic glass spacers to provide precise anodecathode spacing. Results of bench and beam tests of the prototype chambers are discussed. (C) 2001 Elsevier Science B.V. All rights reserved.

\footnotetext{
*Correspondence address. 4004 Wesbrook Mall, Vancouver, BC, Canada V6T 2A3. Fax: +1-604-222-1074.

E-mail address: davydov@triumf.ca (Y. Davydov).
}

\section{Introduction}

The purely leptonic decay $\mu \rightarrow$ ev $\bar{v}$ is an ideal tool to test the Standard Model because the Michel parameters $\rho, \delta, \xi$ and $\eta$ [1] take welldefined values for pure $V-A$ coupling. TRIUMF 


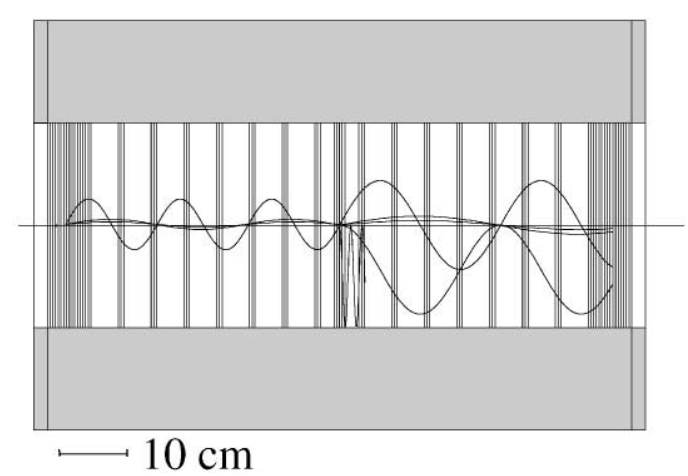

Fig. 1. Schematic of the spectrometer with positron helices.

experiment E614 [2] will measure the entire energy and angle distribution of positrons from the decay of polarized muons and so will improve our knowledge of these parameters by factors of up to 40 , leading to the possible discovery of new physics outside the Standard Model. It will be done using a high-precision symmetric spectrometer with planar drift chambers placed in the center of a uniform $2.2 \mathrm{~T}$ solenoidal magnetic field (see Fig. 1). The layout and the materials of the E614 drift chambers have been chosen to minimize the effect of multiple scattering and energy loss of both the incoming muons and the positrons from muon decay. All field wires have been eliminated. The cathodes are made from double-sided aluminized mylar foil and serve for adjacent chambers as well as gas containment foils. Cool low $Z$ pure dimethylether (DME) has been chosen as the drift chamber gas. The spaces between the modules will be filled with He. As a result the total amount of material from the target to the input window is approximately $15 \mathrm{mg} / \mathrm{cm}^{2}$.

\section{Chamber design and winding equipment}

The chamber modules are comprised of planes with 2 or $4 \mathrm{~mm}$ anode wire pitch. All results presented in this work are for the $4 \mathrm{~mm}$ pitch chambers. These chambers have eighty $15 \mu \mathrm{m}$ diameter gold plated $\mathrm{W}(\mathrm{Re})$ sense wires. The cathode foils are $6.4 \mu \mathrm{m}$ thick double-sided aluminized mylar stretched with a force of up to $0.34 \mathrm{~kg} / \mathrm{cm}$. The anode-cathode gap is $2 \mathrm{~mm}$. A circular glass frame and ceramic glass rings are key parts of the chamber plane. The ceramic glass has an elastic modulus of about $57.0 \mathrm{GPa}$ and temperature expansion coefficient $3 \times 10^{-7} \mathrm{~K}^{-1}$. The thickness of the glass frames is about $3.2 \mathrm{~mm}$. Two glass tables with surface flatness larger than $0.3 \mu \mathrm{m}$ with a set of glass spacers and glass rulers are used for chamber manufacture. The ceramic glass rings, glass spacers and glass rulers are all accurate to $\pm 0.5 \mu \mathrm{m}$. The $340 \mathrm{~mm}$ glass rulers have 330 diamond cut grooves with pitch $1.0000 \pm 0.0015 \mathrm{~mm}$.

The ceramic glass rings and cathode foil are glued into the glass frame with laminated $0.125 \mathrm{~mm}$ thick double-sided printed circuit board (PCB) using the $1.997 \mathrm{~mm}$ thick ceramic glass spacers. This procedure provides a precise positioning of the cathode between the two surfaces of the ceramic glass rings, hence, assuring the anodecathode spacing. Positioning of the wires is controlled by two CCD cameras observing the glass ruler grooves. Each wire is fixed on the PCB with a spot of epoxy and then is soldered to the PCB pads. The average tension of the sense wires is typically $30 \pm 1 \mathrm{~g}$. The position of each wire in the $X$ and $Z$ directions is checked using two CCD cameras. The measured pitch of the wires is $4.000 \pm 0.006 \mathrm{~mm}$. The error includes the inaccuracy of the measuring system. The planes are mounted one after another with wires in alternating $X$ and $Y$ directions using the ceramic glass ring surfaces as reference. A plane without wires serves as the last cathode foil of a complete module. Such an assembly has precise anode-cathode distances in each plane of the module. Modules in the spectrometer have from 2 to 8 wire planes.

\section{Prototype chambers test results}

Several modules containing 2-7 wire planes have been tested. Due to the very small gaps between the glass frames of adjacent chamber planes (about $0.6 \mathrm{~mm}$ ) polyethylene straw tubes are used to bring the gas into the wire region of each chamber. DME gas and $\mathrm{Ar}+$ iso- $\mathrm{C}_{4} \mathrm{H}_{10}$ mixture have been used for bench and beam tests. 
The cathodes are grounded and positive high voltage is applied to the wires. This allows the possibility of setting lower voltages on some planes in case of problems. Also, the breakdown discharge time with a grounded cathode will be longer, hence safer, despite the larger accumulated charge in the system. Our preamplifiers (ASIC [3]) have a gain of $1 \mathrm{mV} / \mathrm{fC}$ and a dynamic range -400 to $+20 \mathrm{fC}$. The signals are fed to a discriminator and then to a 96 channel multihit Fastbus TDC (LeCroy model 1877). Both leading and trailing edges of each signal are recorded for future analysis.

The gas gain has been checked across a plane with an ${ }^{55} \mathrm{Fe} \gamma$-source and revealed to be homogeneous within $\pm 5 \%$. The chambers have an efficiency of 0.999 across almost the entire cell with pure DME for normally incident minimum ionising particles and a threshold of $1.0 \mu \mathrm{A}$. The efficiency plateau is not less than $400 \mathrm{~V}$ wide. Crosstalk between channels is much higher for slow $4 \mathrm{MeV}$ muons as compared to the relativistic positrons. A beam test used $30 \mathrm{MeV}$ protons to simulate the passage of $4 \mathrm{MeV}$ muons. Fig. 2 presents results of this test with pure DME. The top frame shows the distribution of pulse duration from one plane when all events were included. The bottom frame is the same data but events with only a single hit per plane are included. In the latter case one can see that the short signals, which are the crosstalk between adjacent channels, disappear. This provides a method to distinguish off-line the real hits from the corresponding short coincident signals on the adjacent channels. Implementation of ground strips between the signal traces on the PCB, screening of the high-voltage coupling capacitors and the traces on the preamplifier service boards has reduced the crosstalk level to less than $0.5 \%$, a factor of 6 less than when the beam tests shown in Fig. 2 were performed.
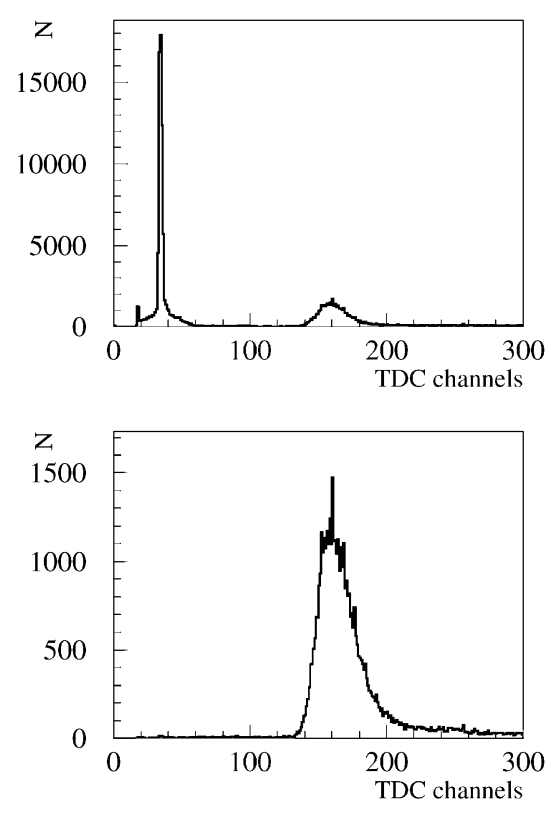

Fig. 2. Signal duration for one plane. Top frame shows all signals per event while bottom one includes single hit events only.

The bench and the beam tests of prototype chambers have proved the chamber design and manufacture procedure are adequate. Quality control during production allows for checks at each step. These chambers have demonstrated high operational stability with pure DME.

\section{References}

[1] L. Michel. Proc. Phys. Soc. A 63 (1950) 514; C. Bouchiat, L. Michel. Phys. Rev. 106 (1957) 170.

[2] E614 Collaboration. Precision measurement of the Michel parameters in $\mu^{+}$-decay, TRIUMF Research Proposal 614, (unpublished); M. Quraan et al., Nucl. Phys. A 663\&664 (2000) 903c.

[3] R.J. Yarema et al., IEEE Trans. Nucl. Sci. NS-39 (1992) 742. 